\title{
ADAPTATION TO DISPLACED AND DELAYED VISUAL FEEDBACK FROM THE HAND ${ }^{1}$
}

\author{
RICHARD HELD, AGLAIA EFSTATHIOU, AND MARTHA GREENE
}

\author{
Massachusetts Institute of Technology
}

\begin{abstract}
S viewed an oscilloscope trace of a short bar which appeared at the position of his nonvisible right hand. The motion of the trace was equivalent to the movements of the hand, which were restricted to a frontal plane. The bar could be optically displaced by 20 diopter prisms, either to the left or right, and its motion could be made to lag behind that of the hand by 1 of 6 delay intervals ranging from 0.0 to $3.0 \mathrm{sec}$. Results show that the adaptation to displacement found with no delay is completely eliminated under all delay intervals, including the minimum of $0.3 \mathrm{sec}$.
\end{abstract}

In recent years investigators have experimented with devices which introduce a time delay between the occurrence of an event and its registration at the eye. Normally, as $S$ moves his hand, motion of its retinal image follows with a negligible lag caused by the transmission time of light. However, the new devices have been used to introduce delays in visual feedback ranging from a fraction of a second up to several seconds. A few authors have reported severe loss of control in self-paced, visually guided movements (Kalmus, Fry, \& Denes, 1960; Smith, McCrary, \& Smith, 1960) as a consequence of delay. However, extensive experiments of Ferrell and Sheridan (Ferrell, 1965; Sheridan \& Ferrell, 1963) have shown that $S$ s will usually adopt a simple strategy of "move and wait" and suffer no such loss of control. Use of this strategy allows time to be traded for accuracy in the performance of manipulative tasks under delay.

Although the appropriate strategy

1 This research has been supported by the National Institute of Mental Health (Grant M-7642), the National Aeronautics and Space Administration (Grant NsG-496), the United States Air Force Office of Scientific Research (AF-AFOSR 354-63), and the Rockefeller Foundation. apparently precludes loss of visual control of manipulation under delay, we have reason to suspect that the delay should have an effect on at least one aspect of eye-hand coordination. A series of experimental studies has demonstrated the role of movementproduced visual feedback in adaptation to various types of sensory rearrangement (Held \& Freedman, 1963; Pick \& Hay, 1965) as well as in the early development of sensorimotor coordination (Held \& Hein, 1963). Because of spatial and kinematic relations, these feedback signals are correlated with output signals to the musculature responsible for the movements that produce the change in retinal stimulation. We have advanced the idea that this correlated information is basic for adaptation to optical displacement produced by wedge prisms and to other forms of rearrangement. Confirming this notion are the findings that when $S$ views his hand optically displaced and with the normal relation between innervation of limb muscle and actual movement of the limb upset by varying external forces, there is a marked reduction of adaptation to the displacement (Abplanalp \& Held, 1965; Efstathiou, 1963).

Delay complicates the normal relation 
between spatial dispositions of the hand and its retinal image. Under delay the position of the retinal image at any given time is related to the past position of the hand as a function of the delay interval and the path and velocity of movement of the hand. If, as we suspect, the nervous system cannot factor out the effects of these variables the result should be equivalent to decorrelation of output and feedback signals. Consequently, adaptation to a constant visual displacement, such as is produced by prism goggles, should be reduced. Comparison of the magnitudes of adaptation to displacement under normal, no delay conditions with those produced under delay should provide a sensitive test of the effect of delay on the vistuomotor control system.

\section{Method}

Apparatus.-During exposure $S$, seated on a stool, firmly grasped a knob at the end of a control stick, 17.5 in. long, whose other end was fixed to a gimbal. Movement of $S$ 's hand was then restricted to the surface of a sphere of $17.5 \mathrm{in}$. radius tangent to his frontal parallel plane at a distance of $14 \mathrm{in}$. from his eyes. The movements were further limited to right-left and up-down components of displacements in this plane of 15 and 11 in., respectively, and rotations around an axis along the length of the control stick. Movements of the stick actuated three potentiometers of which one was responsive to rotation and the other two to the two components of translatory displacement of the hand. (See Appendix for a more complete discription). The potentiometers modulated three voltages which could be fed either directly to an oscilloscope or to the recording heads of a tape transport system. In the latter case, delays of required duration were produced by varying the length of tape between recording and playback heads. Speed of tape transport was constant. The outputs of the playback heads were then fed to the oscilloscope. The voltages actuating the oscilloscope produced rotational and translational displacements of the scope trace (a straight line segment, $1 \frac{1}{2}$ in. long and in. wide) across the scope face. These motions were proportional in magnitude and direction to those of the hand which actuated the system. The scope trace was optically projected onto a ground-glass screen so that its magnified displacements were equivalent to those of the hand. The $S$ viewed the screen in a fully reflecting mirror in an otherwise dark room. The position and orientation of the mirror was such as to make the virtual image of the trace remain superimposed on the invisible hand under conditions of no-delay and no constant visual displacement. For convenience, wedge prisms were used to displace the image of the hand although mechanical or electronic means might have been used.

Before and after exposure, $S$ was tested in a separate apparatus (Held \& Gottlieb, 1958). He marked, with his right hand, the virtual images of two targets viewed binocularly in a fully reflecting mirror that obscured his hand. The targets appeared to lie on a surface whose location approximated that in which his hand moved during exposure. Throughout the experiment, $S$ 's head was held by a bite board.

Subjects.-Male, college undergraduates served as $S \mathrm{~s}$. All were naive as to the object of this experiment.

Procedure.-Each of $24 S$ s was first run in two sessions without time delay. Half of the $S$ s were exposed with base left (BL) prisms first followed by base right (BR) prisms on the next day; the other half were given the reversed sequence. The sequence of events within each session was as follows: (a) Preexposure marking: $S$ alternately marked the apparent position of each of two visual targets for a total of 20 marks, withdrawing his hand between markings. (b) Exposure: $S$ grasped the knob of the apparatus firmly and moved it from left to right and back at $21 \mathrm{c} / \mathrm{min}$ to the beat of a metronome. He was instructed to watch the oscilloscope trace as he viewed it through a pair of 20 diopter prisms oriented either BR or BL, depending on the session. (c) Postexposure marking: After 2, 6, 14, and 30 min. of cumulated duration of exposure to the prisms, $S$ was tested in a manner identical to that of the preexposure marking. From the $24 S$ s tested, we selected 6 who showed significant and approximately equal adaptation under both $\mathrm{BR}$ and $\mathrm{BL}$ exposures. Experiments with each of these $6 S$ s were then conducted in 12 further sessions.

The procedure of each of the next 10 sessions was essentially identical to that of the first 2 except for the fact that in each session $S$ 's visual feedback from his hand movements 
was subjected to a constant time lag whose magnitude was varied from session to session. The delays used were $0.3,0.5,0.9$, 1.7 , and 3.3 sec. The 10 sessions using combinations of each of the five delays with each of the two prism orientations were presented to $S \mathrm{~s}$ in varied sequences. The final 2 sessions were identical to the first 2 in which no delay was introduced.

\section{REsUlts}

Pre- to postexposure shifts for each exposure duration were computed for all delays. The $S$ 's mean shifts, averaged across the two target locations, were computed for all six magnitudes of time lag $(0.0-3.3 \mathrm{sec}$.). The shifts for $\mathrm{BR}$ and $\mathrm{BL}$ conditions were also averaged to yield a single score for each condition of delay. A preliminary anal$y$ sis of variance had shown that $S$ 's shifts during the first two no-delay sessions did not differ significantly from those obtained in the last two no-delay sessions; therefore, the averaged shifts of these four sessions were used for further analysis. The $S$ 's mean shifts are presented in Fig. 1. With time lag in the feedback loop, none of these mean shifts was significantly different from zero by one-tailed $t$ tests at the .05 level. However, with no delay the mean shifts after 2,6 , and 14 min. of cumulative exposure were significant beyond the .01 level of confidence and after $30 \mathrm{~min}$. beyond the .001 level.

An analysis of variance of the mean shifts for all magnitudes of delay indicated that the magnitude of delay and cumulative exposure main effects were highly significant $(p<.001)$. This indicated that, first, the introduction of time delay differentially affected adaptation to displacement, and second, that adaptation to displacement was influenced by the amount of time that $S$ was exposed to prisms.

A second analysis of variance in which the shifts obtained under no-delay sessions were omitted indicated that

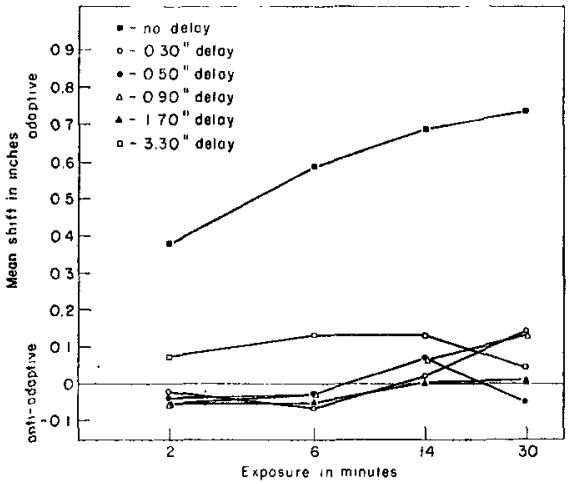

FIG. 1. Cumulated shifts in marking visible targets.

the amount of delay main effect, failed to reach significance at the .05 level implying that amount of delay, ranging from 0.3 to $3.3 \mathrm{sec}$, did not differentially affect the amount of shift resulitng from exposure. The cumulative exposure main effect was significant $(p<.001)$. However, as mentioned above none of the mean shifts reached significance under delay conditions. The general trend of the curves for delayed feedback (Fig. 1) was not consistently in the adaptive direction. Since the interaction between magnitude of delay and cumulative exposure was not significant, the trends of the curves for the several delays do not differ significantly. Consequently, although cumulative exposure entails significant change, the variation is not of the type that we identify with adaptation.

An analysis of variance of the scatter (standard deviation in right-left dimension) in markings of individual target points showed no significant changes over the period of exposure.

\section{ConcLusions}

The results indicate that the correlating mechanism, believed to account for adaptation, cannot handle a feedback signal 
delayed by as little as $0.3 \mathrm{sec}$. Such delayed signals, then, produce one of the effects that we have ascribed to decorrelation. Of great interest are the effects of delays of less than $0.3 \mathrm{sec}$. now under study. Such delays are of the order of magnitude of transmission times in the central nervous system.

\section{APPENDIX}

\section{Electronics of Delayed Feedback Apparatus}

The electronic portion of the apparatus can be described as follows: $S$ 's hand held a control stick which operated a gimbal arrangement consisting of two linear voltage divider potentiometers for $X$ and $Y$ directions of translational movement and a sinecosine potentiometer for rotation (Fig. 2). The two linear potentiometers were fed a balanced dc voltage of $\pm 150 \mathrm{v}$. Any deviation of the control stick from the center position resulted in a voltage corresponding in magnitude and polarity to the magnitude and direction of the stick movement. The sine-cosine potentiometer was fed a $60 \mathrm{cps}$ ac voltage of about $7 \mathrm{v}$. RMS which was used to provide a straight-line trace, rotatable on the oscilloscope screen. The outputs of the sine-cosine potentiometer consisted of two voltages, corresponding in magnitude to the sine and cosine of the rotational displacement of the control stick from a zero position.

The $\sin \theta$ signal was added to $X$ and $\cos \theta$ to $Y$ thereby superimposing rotational on translational information. The output of each adder consisted of a dc voltage varying in polarity and magnitude with a $60 \mathrm{cps}$ ac voltage varying in magnitude superimposed upon it. Each composite signal was then fed into an analog data recorder where it modulated a 7,500 cps carrier and was then recorded on magnetic tape. Each signal was picked up on a multichannel playback head placed a short distance from the recording head, thus introducing a delay into the system. This delay was directly proportional to the distance between the record and playback heads and inversely proportional to the speed of the tape. The tape speed remained constant at $7 \frac{1}{2} \mathrm{in} / \mathrm{sec}$ for the entire experiment. The played-back signal was demodulated and amplified.

DIAGRAM OF DELAY APPARATUS

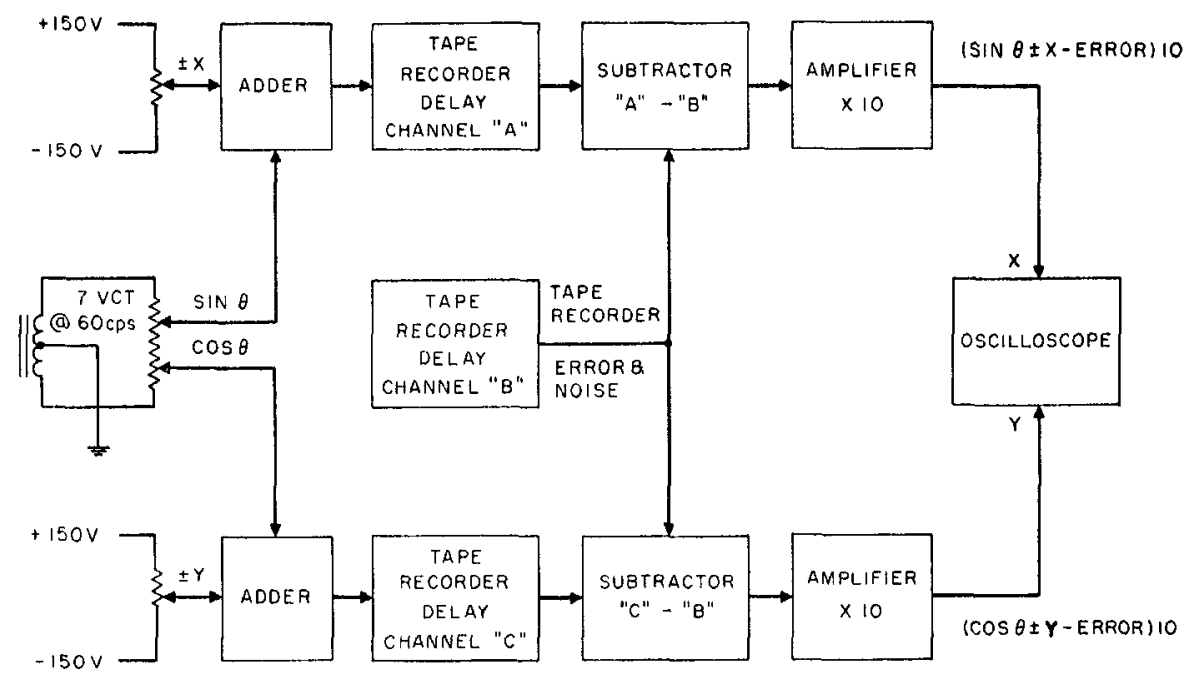

FIG. 2. Block diagram of apparatus used to introduce delay into the hand-eye feedback loop. 
At the same time, another channel of the tape recorder was operated with shorted input to produce a signal corresponding to noise and drift generated by the tape recorder. This error signal was subtracted from the two information channels, thus eliminating any error introduced into the information by the tape recorder. The signals were then amplified 10 times and applied to the vertical and horizontal channels of the oscilloscope.

The adders, subtractors, and amplifiers were made using standard analog computer operational amplifiers with external resistor networks to obtain the proper functions. The modulator/tape recorder/demodulator unit was a Mnemotron Model 200 with a special adjustable idler roller to give several different amounts of delay.

\section{REFERENCES}

Abplanalp, P., \& Held, R. Effects of decorrelated visual feedback on adaptation to wedge prisms. Paper read at Eastern Psychological Association. Atlantic City, N. J., 1965.

Efstathiov, A. Correlated and de-correlated visual feedback in modifying eyehand coordination. Paper read at Eastern Psychological Association, New York, 1963.

FERRELL, W. Remote manipulation with transmission delay. IEEE Trans. hum. Factors Elect., 1965, HFE-6, 24-32.

Held, R., \& Freedman, S. Plasticity in human sensorimotor control. Science, 1963, $142,455-462$.

Held, R., \& Gottlies, N. Technique for studying adaptation to disarranged eyehand coordination. Percept. mot. Skills, $1958,8,83-86$.
Held, R., \& Hein, A. Movement-produced stimulation in the development of visuallyguided behavior. J. comp. physiol. Psychol., 1963, 56, 872-876.

Kalmus, H., Fry, D. B., \& Denes, P. Effects of delayed visual control on writing, drawing, and tracing. Lang. Speech, 1960, 3, 96-108.

Pick, H. L., \& HAY, J. C. A passive test of the Held reafference hypothesis. Percept. mot. Skills, 1965, 20, 1070-1072.

Sheridan, T. B., \& Ferreld, W. R. Remote manipulative control with transmission delay. IEEE Trans. hum. Factors Electron., 1963, HFE-4, 25-29.

Smith, W., McCrary, J., \& Smith, K. Delayed visual feedback and behavior. Science, 1960, 132, 1013-1014.

(Received October 13, 1965) 